\title{
Procedures for the relative calibration of the SiPM gain on ASTRI SST-2M camera
}

\author{
D. Impiombato, O. Catalano, \\ S. Giarrusso, T. Mineo, G. La Rosa, \\ C. Gargano, P. Sangiorgi, A. Segreto, \\ G. Sottile, G. Bonanno, S. Garozzo, \\ A. Grillo, D. Marano, G. Romeo, \\ R. Gimenes
}

Received: date / Accepted: 11/11/2016

\begin{abstract}
ASTRI SST-2M is one of the prototypes of the small size class of telescopes proposed for the Cherenkov Telescope Array. Its optical design is based on a dual-mirror Schwarzschild-Couder configuration, and the camera is composed by a matrix of monolithic multipixel silicon photomultipliers managed by ad-hoc tailored front-end electronics. This paper describes the procedures for the gain calibration on the ASTRI SST-2M. Since the SiPM gain depends on the operative voltage and the temperature, we adjust the operative voltages for all sensors to have equal gains at a reference temperature. We then correct gain variations caused by temperature changes by adjusting the operating voltage of each sensor. For that purpose the SiPM gain dependence on operating voltage and on temperature have been measured. In addition, we present the calibration procedures and the results of the experimental measurements to evaluate, for each pixel, the parameters necessary to make the trigger uniform over the whole focal plane.
\end{abstract}

Keywords Front-End; ASIC for SiPM; ASTRI; CTA

D. Impiombato $(\square)$

INAF, Istituto di Astrofisica Spaziale e Fisica cosmica di Palermo, via U. La Malfa 153 I-90146 Palermo, Italy Tel.: +39 0916809468 Fax: +39 0916882258

E-mail: Domenico.Impiombato@iasf-palermo.inaf.it

O. Catalano, S. Giarrusso, T. Mineo, G. La Rosa, C. Gargano, P. Sangiorgi, A. Segreto, G. Sottile

INAF, Istituto di Astrofisica Spaziale e Fisica cosmica di Palermo, via U. La Malfa 153, I-90146 Palermo, Italy

G. Bonanno, S. Garozzo, A. Grillo, D. Marano, G. Romeo

INAF, Osservatorio Astrofisico di Catania, via S. Sofia 78, I-95123 Catania, Italy

R. Gimenes

Instituto de Astronomia, Geofísica e Ciências Atmosféricas, Universidade de São Paulo Rua do Matão 1226, 05508-090 São Paulo, SP, Brazil 


\section{Introduction}

The use of Imaging Atmospheric Cherenkov Telescopes (IACTs) in gamma-ray astronomy opened a new frontier in the study of the emission from high-energy sources such as supernovae, neutron stars and supermassive black-holes. Since the discovery of $\mathrm{TeV}$ emission from the Crab nebula with the Whipple $10 \mathrm{~m}$ [1] in 1989, the number of known sources emitting in this range of energy has rapidly increased to hundreds in a relatively small number of years. The detection of Very High Energy (VHE) gamma photons is possible by observing the Cherenkov light produced by the relativistic secondary particles in the air shower generated by the interaction with the Earth's atmosphere. This light, emitted within a cone of $\sim 1.3^{\circ}$ in the ultraviolet band, reaches the ground in a "pool" with $\sim 120$ m radius. It is very faint and lasts only a few ns with a duration that depends mainly on the distance between the shower core and the telescope axis [2].

Significant progress on the investigation of VHE gamma-ray phenomena has been achieved in the last years with the development of more complex IACT instruments such as H.E.S.S. [3], MAGIC [4] and VERITAS [5] which represent the current state-of-the-art. Nevertheless, lots of puzzles still remain in the understanding of different features of the TeV gamma-ray astronomy. So, the international $\mathrm{TeV}$ astrophysics community is moving towards a newer generation of IACT telescopes that will form the Cherenkov Telescope Array (CTA) observatory [6].

CTA will be the largest instrument for gamma-ray astronomy ever conceived and will comprise all advantages of the IACT technique. The CTA observatory will consist of an extended array of more than one hundred of telescopes of different sizes (large, medium, small) deployed over two sites, one in each hemisphere. At its southern site, CTA plans to install about 70 small size telescopes (SSTs), dedicated to study the sky from a few TeV up to hundreds of $\mathrm{TeV}$. The deployment of the telescopes will be done at different times, along intermediate steps. At the beginning, during the so-called CTA pre-production phase, small subset of telescopes will be installed to assess readiness for mass production. At the CTA Southern site, during the preproduction phase, it is foreseen the deployment of the ASTRI mini-array led by Italian National Institute of Astrophysics (INAF) in synergy with the Universidade de São Paulo (Brazil) and the North-West University (South Africa). The ASTRI mini-array $[7,8]$ will be composed of nine dual-mirror small size telescopes whose end-to-end prototype, named ASTRI SST-2M [9] and developed by INAF, has been recently inaugurated in Italy. Several challenging but innovative technological solutions characterize the ASTRI concept [10]: the telescope adopts a dual-mirror (2M) Schwarzschild-Couder optical design [12] and the camera at the focal plane is composed of a matrix of Silicon photomultipliers (SiPMs) managed by an appropriate front-end electronics [13]. The telescope design is based on a focal ratio of 0.5, leading to an equivalent focal length of $2.15 \mathrm{~m}$ and an average effective collecting area of about $6.5 \mathrm{~m}^{2}$ with a resulting full field of view, FoV, of $\sim 9.6^{\circ}$. 
This paper describes the procedures and the lab measurements necessary for the ASTRI SST-2M SiPMs gain calibration. The main purpose of these procedures is to keep during the normal data taking the gain uniform and stable without the aid of instrumentation external to the camera, correcting for variation compared to the nominal values of the involved parameters (relative calibration). These objectives are reached providing the camera with a set of reference values for operative voltage and with some coefficients to correct gain variations. Measurements aimed to determine the values of the gain variation as a function of the operative voltage and temperature are presented in this paper together with the level of accuracy due to different sources of errors.

In addition, we present the calibration procedures and the results of measurements performed in lab to evaluate, for each pixel, the parameters for making uniform the trigger over the whole focal plane.

\section{ASTRI Camera}

The ASTRI SST-2M camera has a truncated-cone shape and its overall dimensions are $665 \mathrm{~mm} \times 665 \mathrm{~mm} \times 515 \mathrm{~mm}$, including mechanics and interfaces with the telescope structure, for a total weight of $\sim 70 \mathrm{~kg}$. An exploded $3 \mathrm{D}$ visualization of the camera assembly is shown in Figure 1. The focal surface is composed by a matrix of SiPM Hamamatsu Silicon Photomultiplier S11828-3344M [14] working at an operative voltage of $\sim 70 \mathrm{~V}$. Each SiPM sensor is formed by $4 \times 4$ squared physical pixels, $3 \mathrm{~mm} \times 3 \mathrm{~mm}$, made up of 3600 single-photon avalanche diode (SPAD) of $50 \mu \mathrm{m}$ pitch [15].

In order to match the angular resolution of the optical system, the physical pixels of each sensor unit are grouped $2 \times 2$ in the so-called camera pixel $(6.2 \mathrm{~mm} \times 6.2 \mathrm{~mm})$ having a sky-projected angular size of $0.17^{\circ}$. The aggregation of $4 \times 4$ sensor units $(8 \times 8$ camera pixels $)$ forms a Photon Detection Module (PDM), and 37 PDMs form the camera (see Figure 2). This modular design allows to better follow the curvature of the focal plane and makes easier the maintenance of small portions of the camera being the PDMs physically independent.

In order to protect the camera sensors from the external atmospheric environment, an optical-UV transparent Poly Methyl MethAcrylate (PMMA) window is mounted onto the focal surface support structure covering all the PDMs. This window is modeled with the same radius of curvature of the focal surface (see Sect. 2.1). A side emitting Fiber Optics system(FOC) is located along the PMMA circumference. It is illuminated by a Light Emitting Diode (LED) system and used for the on-field relative calibration of the gain. Its light uniformly illuminates the PDMs units at a controlled intensity.

The ASTRI SST-2M camera is equipped also with a light-tight lid composed of two petals mounted onto the backbone structure of the camera. This prevents accidental sunlight exposure of the focal surface detectors, catastrophic in case of direct light reflected by the mirrors. 


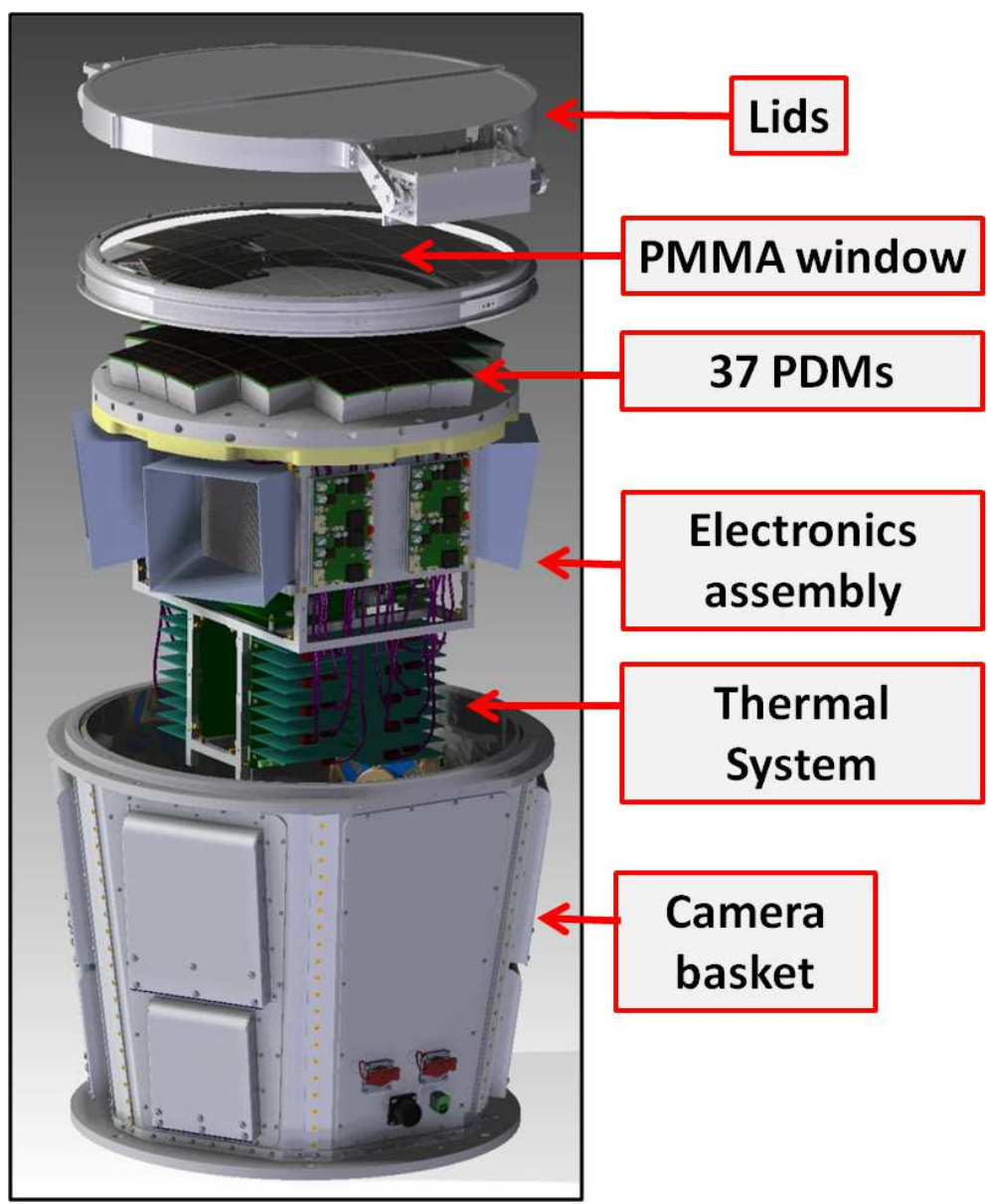

Fig. 1 Exploded view of the Camera body assembly.

The camera is thermally controlled to keep the working temperature on the focal plane within the range $13-17^{\circ} \mathrm{C}$ giving a gain accuracy compliant with the CTA calibration requirements of $\sim 10 \%$ [11]. The temperature of each PDM is monitored by a system of sensors embedded in the SiPM board.

\subsection{Focal plane}

The Focal Surface Camera (FSC), as shown in Figure 1, has a convex-shaped structure with a curvature radius of $1060 \mathrm{~mm}$. PDMs, that are square flat modules, are symmetrically placed on the FSC with angles respect to the telescope axis opportunely chosen in order to follow the curvature of the focal plane. Each PDM, composed of $4 \times 4$ side-by-side SiPMs, is installed on 


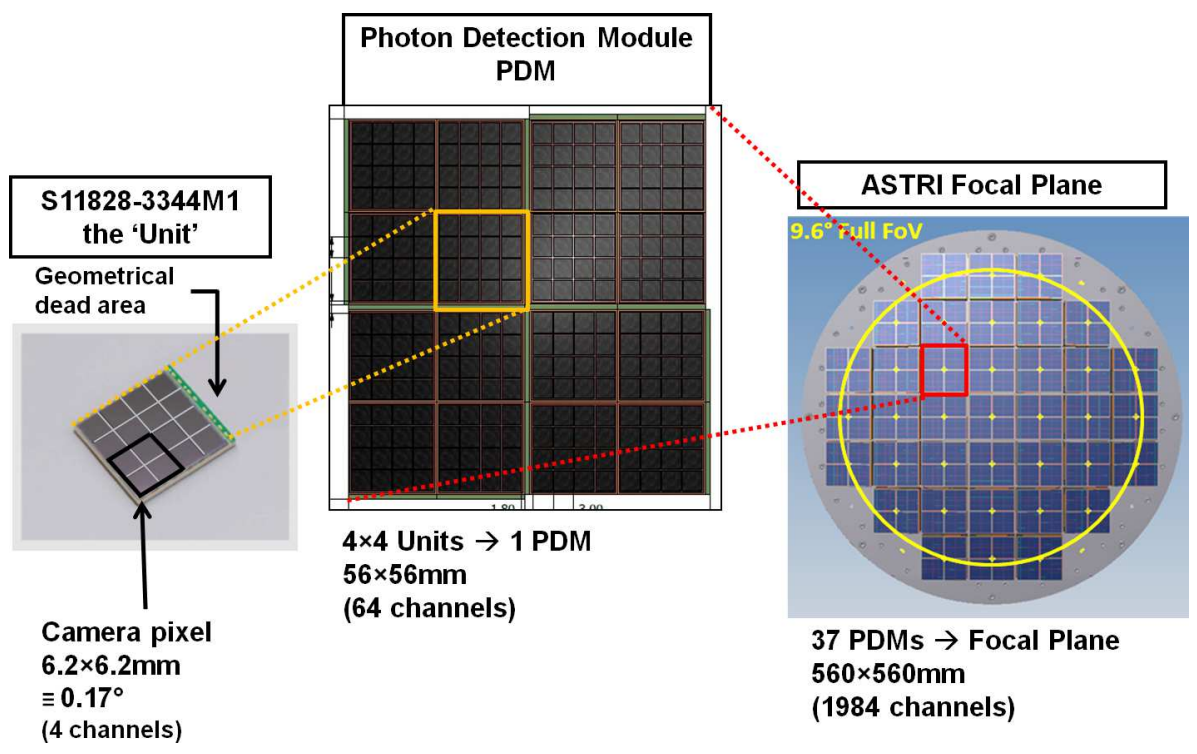

Fig. 2 The black square in the left figure shows one of the camera pixels obtained grouping $2 \times 2$ physical pixels. The central figure shows one ASTRI SST-2M PDM composed of $4 \times 4$ SiPMs. The right figure represents how the PDMs are arranged on the Focal Surface Camera

a Printed Circuit Board (PCB) of the same dimension. The PCB hosts the Front-End Electronics (FEE) composed of two ASICs for the signal read out, four Analogue-to-Digital Converters (ADC) and one Field Programmable Gate Array (FPGA). This FEE-FPGA manages the ASICs slow control, the generation of local trigger and the data analog-to-digital conversion. The Back-End Electronics (BEE) is composed of two main parts that are a Processing System (PS) and a Programmable Logic (PL). It is the main elaboration unit of the camera which controls and manages the overall system, including data, and all ancillaries used to perform operations as the camera thermal regulation, the Voltage Distribution management and the time events stamping. The BEE also provides the functions necessary to process and transmit the event data as obtained by the FEE to an external Data Acquisition Workstation responsible for receiving and storing the data packets.

\subsection{Signal processing}

A dedicated FEE has been designed to catch the very fast pulses of Cherenkov light and also to provide auto-trigger capability. The electronics adopted for the ASTRI SST-2M telescope prototype is the Cherenkov Imaging Telescope Integrated Read Out Chip (CITIROC [16]), INAF design intellectual property. The CITIROC chip is equipped with 32 channels capable of measuring 
charges from 1 to 2000 photoelectrons, assuming a SiPM gain of $10^{6}$. A detailed characterization of the front-end electronics is given in [17]. Two CITIROC devices are devoted to process the SiPM detector signals of each single PDM. Figure 3 represents a schematic view of the whole signal processing path in any CITIROC channel.

The wide dynamic range necessary for the camera read-out is provided by CITIROC using two separated chains integrated in the chip: the High Gain (HG) and the Low Gain (LG).
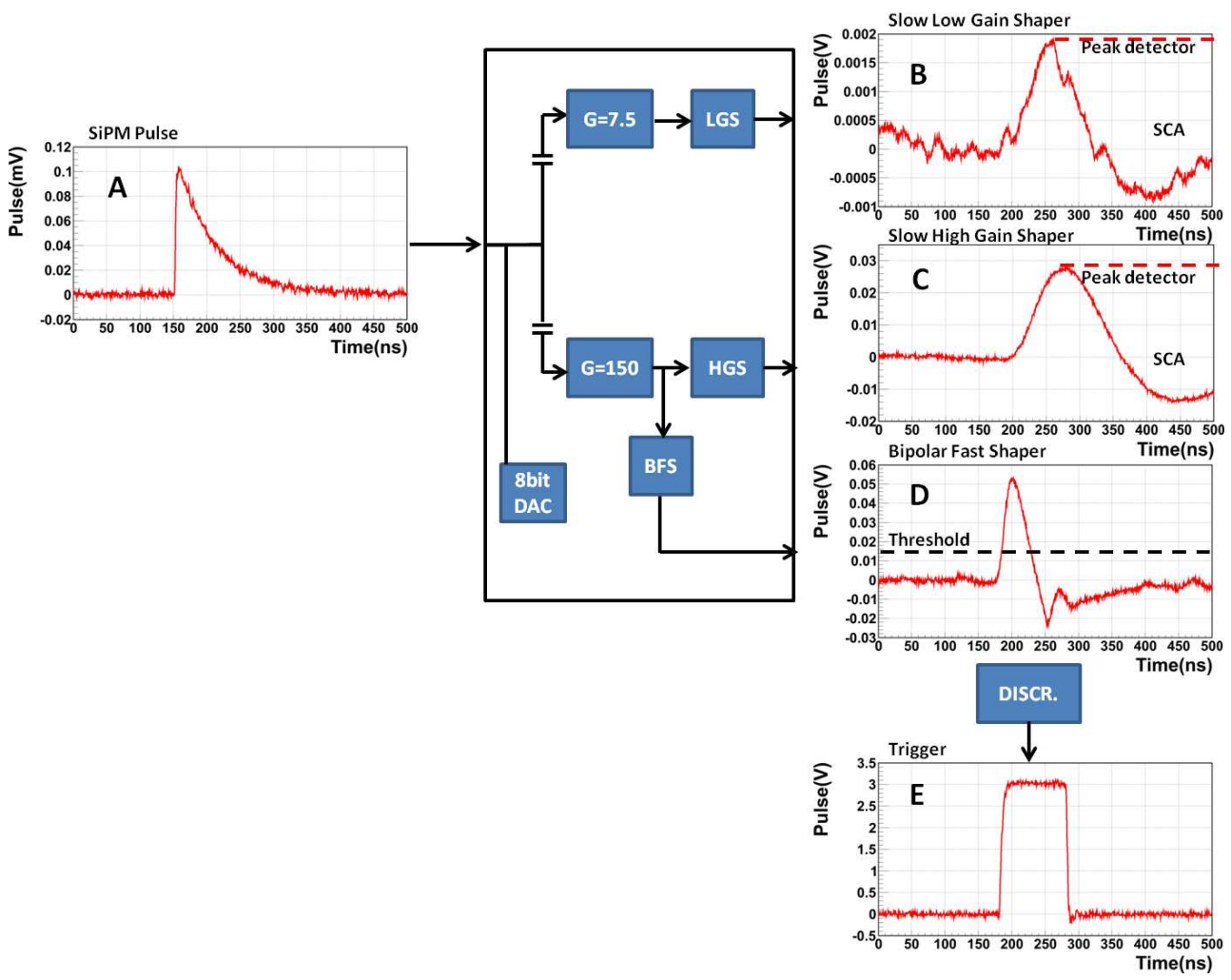

Fig. 3 Signal processing path of one CITIROC channel.

In any CITIROC channel an 8-bit digital to analogue converter (DAC) is integrated, by varying the operative voltage of the corresponding camera pixel. The pulse generated by the SiPM (curve A) is amplified by the two programmable preamplifiers (HG and LG) and then shaped with a constant shaping time of $37.5 \mathrm{~ns}$ by two electronic circuits (HG and LG). Two operation modes are implemented in the HG and LG chains of the ASICs (curves B and $\mathrm{C}$ in Figure 3): the peak detector and the Switched Capacitor Array (SCA). 
The peak detector mode, that is the base-line operating approach, provides the maximum of the shaped pulse height, whereas the SCA mode returns the level of the shaped pulse height at a sampling time delayed from the trigger by a fixed interval. This delay, that depends on the shaping time, is set in order to get the maximum slow shaper signal. In both modes, the conversion of the height of the shaped pulse to the integrated charge introduces a systematic error lower than $1 \%$.

A Bipolar Fast Shaper (BFS) connected to the high gain preamplifier produces a fast signal (curve D) with a shaping time of $\sim 15$ ns. It is connected to a discriminator with a 10 bit programmable threshold. The output of the discriminator gives a digital trigger (curve E) if the signal exceeds the threshold level. Digital triggers are available on the $32+32$ output pads of the two ASICs. A local trigger is activated when $n$ contiguous pixels within a PDM present a signal above the discriminator threshold. This signal is then propagated to all PDMs to start the acquisition of an event in all the focal plane. The number $n$ of contiguous pixels, programmable from 2 to 7 and the trigger threshold, also programmable ( $\geq 4 \mathrm{pe}$ ) depending on the Night Sky Background (NSB), are chosen in order to have a maximum rate of $\sim 600$ $\mathrm{Hz}$ from the whole camera, with a dead time $<3 \%$, well inside the maximum fraction of the required dead time by CTA [18].

The signal outputs are read and converted to digital counts by external ADC devices, at the occurrence of a trigger condition. A Xilinx Artix 7 FPGA governs and controls all the input/output operations from/to CITIROCs and SiPMs.

\section{Relative calibration plan}

The procedure for the relative calibration of the gain adopted in ASTRI SST$2 \mathrm{M}$ camera foresees different steps:

- Define the nominal values of the configuration parameters as temperatures, gains, operative voltages, etc.;

- Determine the parameters of the gain linear variations with operative voltage and temperature;

- Evaluate the level of accuracy for the gain.

When the camera is mounted on the telescope, these results are used to check and correct the gain relatively to the nominal values. These corrections are performed in real time, taking into account the measurements of the embedded thermal sensors.

In addition, further measurements are periodically carried out using the FOC whose data are used during normal data analysis for off-line corrections of the gain.

All the calibration parameters and procedures will be stored and implemented in the BEE. 


\subsection{Gain of SiPM camera pixel}

The SiPM output signal is produced as a result of an avalanche multiplication process characterized by the gain $G$, defined as the number of electrons generated in response to a photon absorption or a thermally generated ignition in the silicon lattice. In Geiger-mode operation, the multiplication factor of an avalanche discharge is expected to grow linearly with the operative voltage according to:

$$
G=\frac{Q_{T O T}}{q}=\frac{C_{p i x e l}\left(V_{o p}-V_{B D}\right)}{q}
$$

where $Q_{T O T}$ is the total charge generated by a single avalanche, $C_{\text {pixel }}$ is the overall pixel capacitance, $q$ is the electron charge, $V_{o p}$ is the operative voltage and $V_{B D}$ is the detector breakdown voltage.

The gain is a function of the differences between the two voltages $V_{B D}$ and $V_{o p}$. The former is a characteristic of the SiPM physical pixel and is mainly sensitive to temperature; the latter is different in each camera pixel and can be opportunely varied to keep the gain uniform over the camera surface and stable in time against temperature variations in $V_{B D}$. In our case, grouping four physical pixels in one camera pixel and applying a single operative voltage introduces an intrinsic spread in the gain.

Any gain variation $\Delta G\left(V_{o p}, T\right)$ can be written in terms of variations of the operative voltage $\Delta V_{o p}$ and of the temperature $\Delta T$ with the following equation:

$$
\Delta G\left(V_{o p}, T\right)=\frac{\partial G}{\partial V_{o p}} \Delta V_{o p}+\frac{\partial G}{\partial T} \Delta T
$$

where $\partial G / \partial V$ is the gain derivative with respect to the voltage variation, and $\partial G / \partial T$ is the gain derivative with respect to the temperature.

For small variations of the temperature and of the operative voltage, $V_{o p}$, $\partial G / \partial V$ and $\partial G / \partial T$ can be considered constant in a first approximation, and the gain can be computed through the following formula:

$$
G(V, T)=c_{1} \cdot \Delta V_{o p}+c_{2} \cdot \Delta T+c_{3}
$$

where $c_{1}=\partial G / \partial V, c_{2}=\partial G / \partial T$, and $c_{3}=G\left(V_{0}, T_{0}\right)$ is the reference gain at a given operative voltage and temperature. The reference gain suggested by manufacturer is $7.5 \times 10^{5}$. However, we assume $c_{3}=6.0 \times 10^{5}$ as the reference gain for all camera pixels and $T_{0}=15^{\circ} \mathrm{C}$ as the reference temperature. Our choice is mainly driven by the need to reduce the cross talk to a value of $12 \%$.

The reference operative voltage $V_{0}$ is set for each camera pixel in order to obtain the reference gain. The average value of $V_{o p}-V_{B D}$ (overvoltage) is $\sim 1.5 \mathrm{~V}$.

To keep $G(V, T)$ constant we have to compensate for the temperature variations by changing $V_{o p}$ according to the relation:

$$
\Delta V_{o p}=-\frac{c_{2}}{c_{1}} \cdot \Delta T
$$




\subsection{CITIROC DAC calibration}

The operative voltage $V_{o p}$ applied to each camera pixel is the sum of the external voltage $V_{\text {ext }}$ and the voltage applied from the input programmable DAC implemented in the CITIROC front-end, $V_{D A C}$, whose dynamical range is $\sim 4.5 \mathrm{~V}$ :

$$
V_{o p}=V_{e x t}-V_{D A C}
$$

In the CITIROC ASIC, the $V_{D A C}$ is linearly dependent on the DAC code according to the following equation:

$$
V_{D A C}=a \cdot D A C+b
$$

where $a$ and $b$ are characteristic of each channel, and then at variance with $c_{1}$ and $c_{2}$ must be measured for all camera pixels.

$V_{D A C}$ can be used to adjust the SiPM gain, compensating for temperature variations. Combining equations (5) and (6), the formula in (4) can be written in terms of $V_{D A C}$ to obtain the following relation implemented in the BEEFPGA:

$$
\Delta D A C=-\frac{c_{2}}{c_{1}} \cdot \frac{\Delta T}{a}
$$

\subsection{Temperature}

The calibration of the temperature sensors embedded in each SiPM board is an essential prerequisite for the SiPM gain equalization. Each SiPM PCB has 9 temperature sensors symmetrically spaced on the rear side of the board. They operate in the range from $-40^{\circ} \mathrm{C}$ to $+125^{\circ} \mathrm{C}$ with a precision of $\pm 0.2^{\circ} \mathrm{C}$. These sensors are constantly monitored by the FEE-FPGA, and the temperatures measured in each PDM are sent to the BEE in order to adjust the gain of the pixels. The sensors are read at a rate of few $\mathrm{Hz}$, adequate for the expected slow-time temperature variations inside the camera. Only the central sensor at the bottom of the board has been absolutely calibrated using a temperature sensor and an appropriate controller provided by LakeShore ${ }^{1}$. The calibration of the other eight sensors relatively to the central one will be accomplished in lab during the functional tests of each PDM.

\section{Laboratory Experimental Set-Up}

All lab measurements have been carried out in the laboratory of IASF-Palermo and OACT-Catania. For convenience, all measurements were performed using an evaluation board containing one CITIROC. Two different sets of measurements were performed:

\footnotetext{
1 http://www.lakeshore.com/Documents/LSTC_325_l.pdf
} 
- the SiPM pixels were illuminated by a Blue Light Diode (B-LED) driven by a pulse generator at a constant rate of $10 \mathrm{kHz}$. The schematic overview is shown in Section $A$ of Figure 4. This set-up was used for the $c_{1}$ and $c_{2}$ evaluation. The gain relative to each measurements was obtained from the average distance between the peaks in the Pulse Height Distributions (PHDs) as explained later on, in Sect. 5.3. The value of $c_{1}$ is obtained keeping the temperature constant and varying $V_{o p}$ in the operating range in steps of $100 \mathrm{mV}$. The coefficient $c_{2}$ is measured keeping the operative voltage to the reference value $V_{0}$ and varying the temperature in steps of about $1{ }^{\circ} \mathrm{C}$ from $13^{\circ} \mathrm{C}$ to $17^{\circ} \mathrm{C}$.

- An arbitrary generator was used to create an waveform signal input signal as similar as possible to the $\mathrm{SiPM}$ one (rise time of a few ns, recovery time of $175 \mathrm{~ns}$ and 1pe amplitude). The input signal was injected in the channels of the CITIROC evaluation board, as shown in in Section $B$ of the Figure 4. These measurements were used to calculate the ADC photoelectron conversion factor from the obtained PHDs (see Sect. 5.3).

- a Keithley high impedance multimeter (not shown Figure 4) was used to evaluate the coefficients $a$ and $b$ of the linear relation DAC voltage-DAC codes (see Sect. 5.2).
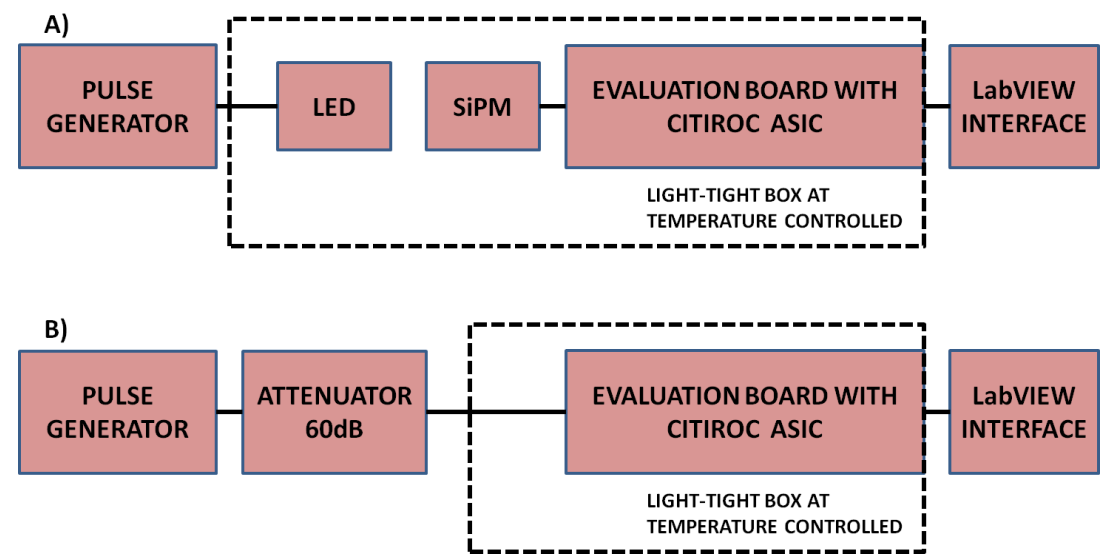

Fig. 4 Experimental set-up. A light-tight box coupled with a Peltier Cooling/Heating system assures a constant temperature on the SiPM under test with stability of $\sim 0.1^{\circ} \mathrm{C}$.

The laboratory equipment includes a signal generator Agilent 81160A plus a precise attenuator to be used with the pulse signal. The CITIROC evaluation board is driven by a Lab-VIEW software ${ }^{2}$ interface provided by Weeroc ${ }^{3}$.

\footnotetext{
2 http://www.ni.com/labview/i/

3 http://www.weeroc.com/
} 


\section{Results}

In the following subsections, results from lab measurements relative to a single camera pixel, taken as example, are reported.

\subsection{Calibration of the reference voltage $V_{0}$}

The reference values of the operative voltage $V_{0}^{p p}$ for each physical pixel, for a gain of $7.5 \times 10^{5}$ at the temperature of $25^{\circ} \mathrm{C}$, were provided by Hamamatsu. These values were verified on a sample in our lab. The values are distributed in the range $71-73.5 \mathrm{~V}$ as shown in the histograms of the left panel of Figure 5. However, grouping four physical pixels in one camera pixel and adopting a single operative voltage $V_{0}$ introduces a spread in the gain. This was evaluated measuring the difference $\left(\Delta V_{0}\right)$ between the minimum and the maximum $V_{0}^{p p}$ of the four physical pixels. The histogram of this spread is shown in the right panel of Figure 5.

This average gain uncertainty can be computed using the mean value of $\Delta V_{0}(29.84 \mathrm{mV})$ and the parameter $c_{1}$, presented in Sect. 5.3. This corresponds to a gain variation of $1.5 \%$ of the Hamamatsu nominal gain $\left(7.5 \times 10^{5}\right)$.
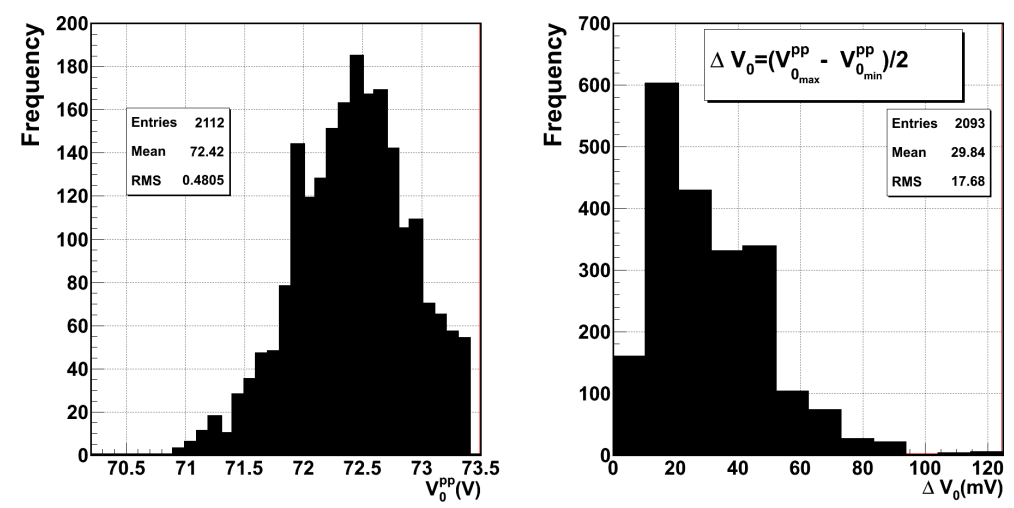

Fig. 5 Histogram of the measured $V_{0}^{p p}$ of all physical pixels (left panel) and of the $\Delta V_{0}$ of the camera pixels (right panel). 


\subsection{Determination of $a$ and $b$ coefficients}

The coefficients of the linear fit of the DAC voltage as a function of the DAC codes are obtained reading the output voltage of the DAC by a Keithley high impedance multimeter, and varying progressively the DAC codes. Figure 6 shows the DAC voltage as a function of the DAC codes for one of the channels.

Each channel has a slightly different slope, so this measurement is required for all camera pixels. The histograms of $a$ and $b$ coefficients are shown in Figure 7 in the left and right panel, respectively.

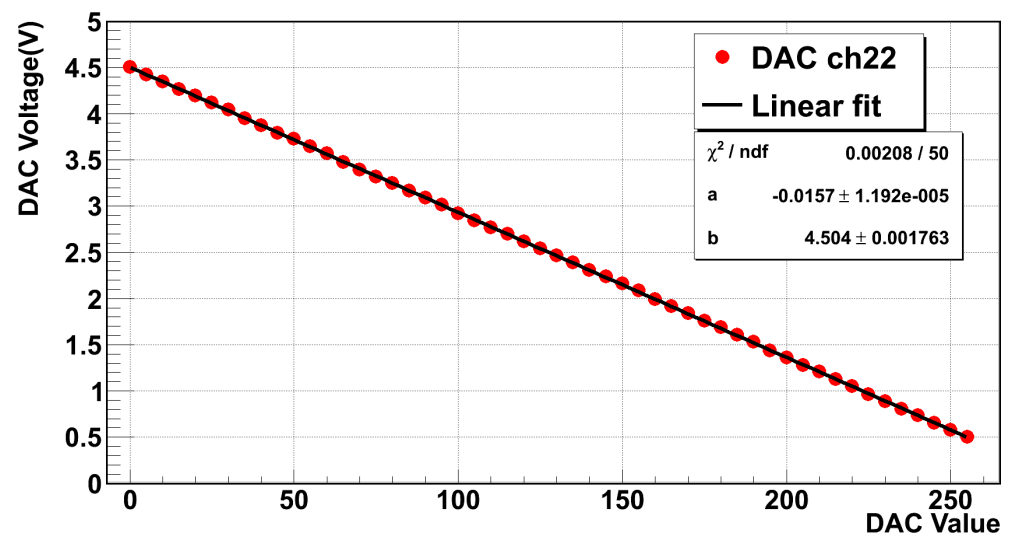

Fig. 6 CITIROC evaluation board DAC voltage as a function of the DAC code for one of the channels. The error of each point is less than $1 \%$, so it is not visible in the figure.
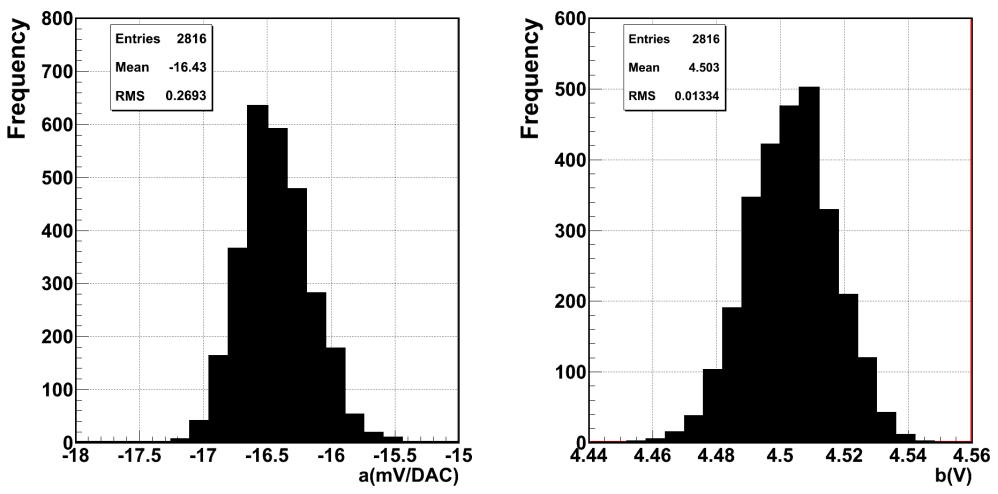

Fig. 7 Histograms of $a$ and $b$ coefficients for all PDM 
The values of $V_{o p}$ can be linearly adjusted channel by channel in the interval $0 \mathrm{~V} \div 4.5 \mathrm{~V}$. This dynamical range is larger than the spread in the values of $V_{0}^{p p}$ $(2.5 \mathrm{~V})$, as shown in the left panel of Figure 5, and larger than the corrections needed for compensating temperature variations $\left(71 \mathrm{mV} /{ }^{\circ} \mathrm{C}\right.$ equivalent to $\sim 4$ $\left.\mathrm{DAC} /{ }^{\circ} \mathrm{C}\right)$.

The average value of $a$ that is the DAC input resolution, corresponds to a gain variation of $6.32 \times 10^{3}$ that is $1.1 \%$ of the nominal gain.

\subsection{Determination of $c_{1}$ and $c_{2}$ coefficients}

The coefficients of the gain variations $c_{1}$ and $c_{2}$ are determined with the PHD pulsing a blue LED at a constant rate of $10 \mathrm{kHz}$ and a time-on duration of 11.5 ns. An example of a PHD at a fixed voltage $V_{0}=71.8 \mathrm{~V}$ and the temperature of $15^{\circ} \mathrm{C}$ is shown in Figure 8 .

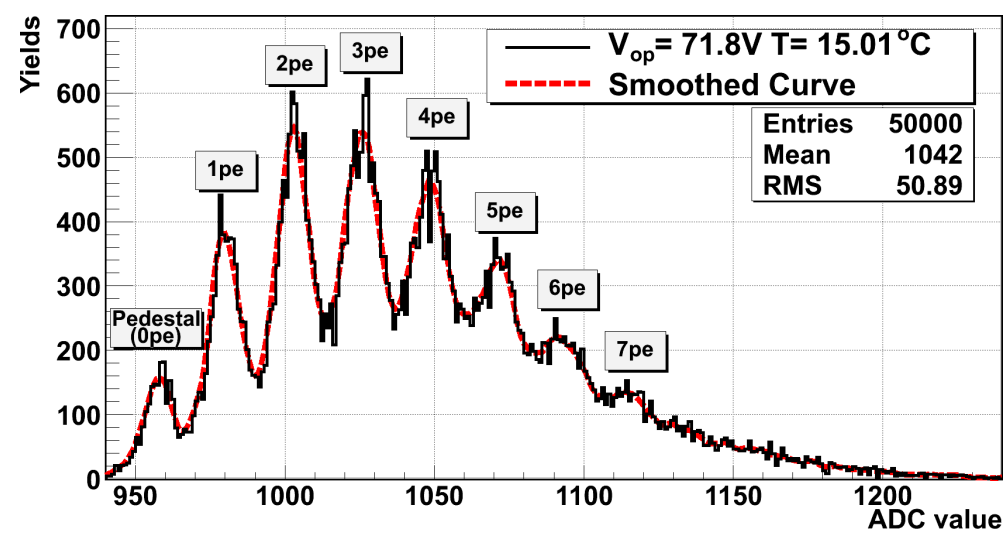

Fig. 8 CITIROC: PHD at a fixed temperature of $15^{\circ} \mathrm{C}$ and $V_{0}=71.8 \mathrm{~V}$ for one of the channels.

The ADC photoelectron conversion factor $p e_{e q}$ is evaluated averaging all distances of subsequent local maxima of the smoothed PHD collected from each SiPM camera pixel. This smoothing is performed with a LOWESS (Locally Weighted Scatterplot Smoothing) method. The $p e_{e q}$ is then computed applying the following equation:

$$
p e_{e q}(A D C)=\frac{1}{N-2} \cdot\left(A D C_{N}-A D C_{2}\right)
$$

where $A D C_{N}$ is the $\mathrm{ADC}$ value corresponding to the last peak and $p e_{e q}$ is the photoelectrons equivalent number in ADC units. Note that the pedestal $(N=1)$, whose position is biased by the electronic noise, is not considered. Its distance from the 1 pe peak distribution has been found significantly different 
from the average distances between the other peaks in updated versions of sensor [20]. We decided to exclude it from computation of the gain in order to implement a procedure that can be exported to newer SiPMs even if for the sensor adopted in the present paper the pedestal could be coherently included in the computation.

The measured $p e_{e q}$ value is then converted in gain comparing it with the value $p e_{r e f}$ obtained by injecting a charge with a pulse shape similar to the $\mathrm{SiPM}$ one corresponding to 1 pe at the reference gain, and applying the following formula:

$$
G\left(V_{o p}, T\right)=\frac{p e_{e q}(A D C)}{p e_{r e f}(A D C)} \cdot G\left(V_{0}, T_{0}\right)
$$

This method allows a finally accuracy on the gain lower than $1 \%$ over a 50000 event acquisition.

We measured the values of $c_{1}$ for one of the camera pixel obtained from PHDs keeping the temperature constant and varying $V_{o p}$ in the operating range in steps of $100 \mathrm{mV}$. The measured gain is then fitted to a linear function of the operating voltage, in which $c_{1}$ is the slope of the best fit line. In Figure 9, an example of a gain plot versus $V_{o p}$ for a camera pixel is shown. The range of the operative voltage is $71.60-72 \mathrm{~V}$, and the gain increases with voltage with the coefficient $c_{1}$ equal to $14.76 \pm 0.78$ (ADC/V).

The coefficient $c_{2}$ is obtained from PHDs keeping the operating voltage to the reference value $V_{0}$ and varying the temperature in steps of about $1{ }^{\circ} \mathrm{C}$ from $13^{\circ} \mathrm{C}$ to $17^{\circ} \mathrm{C}$. Again, fitting the measured gains to a linear function, the value of $c_{2}$ corresponds to the slope. In the example plotted in Figure 10, $c_{2}$ is $-1.06 \pm 0.09\left(A D C /{ }^{\circ} C\right)$. In this case the gain decreases linearly with temperature.

Considering that, in a first approximation, $c_{1}$ and $c_{2}$ are characteristic of the type of SiPM [19], we assumed a single value for all camera pixels. Discrepancies in the gain uniformity are corrected off-line using the FOC as already mentioned in the Sect. 2.

\section{CITIROC trigger calibration}

In addition to the gain calibration we performed in lab the calibration of the camera pixel trigger. This has the aim to uniform the trigger response over all camera. CITIROC is equipped with a 10bit DAC common to all the channels for setting the value of each discriminator voltage. As for any multi-channel electronics front-end, small differences between trigger channels are expected. Performing trigger equalization between pixels is essential for a stable and reliable camera operation. To compensate the channel-by-channel trigger nonuniformity, a 4bit DAC fine-tunes the discriminator threshold level. The linear relation between the 10bit DAC and the 4bit DAC is different for each channel. The resulting set of $324 \mathrm{bit} \mathrm{DAC}$ codes are stored in the registers of each CITIROC providing trigger level discrimination uniformity. 


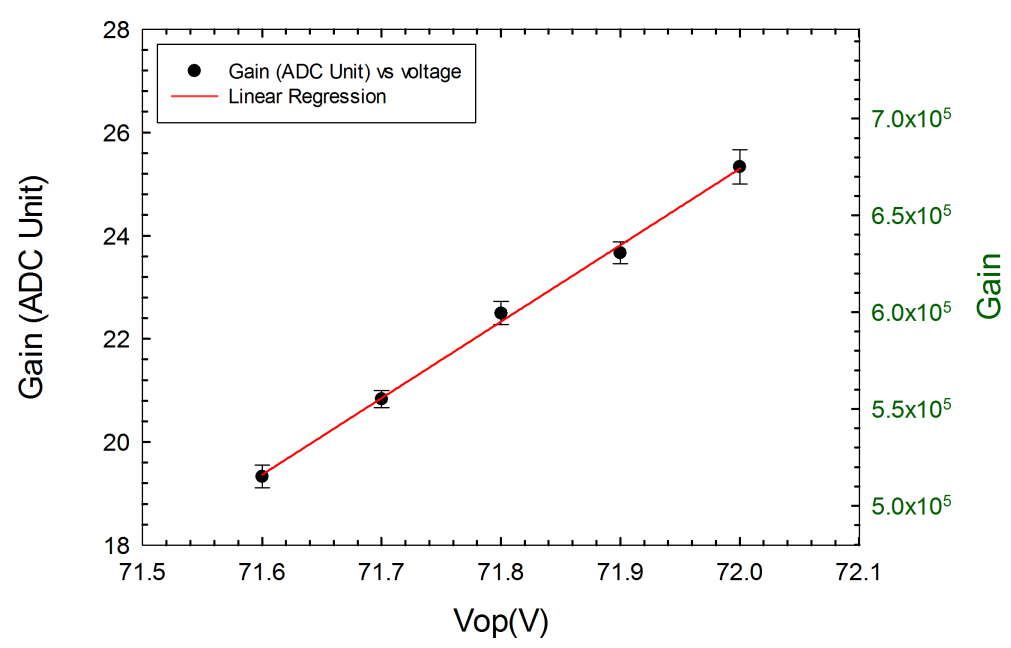

Fig. 9 Determination of the gain variation coefficient in ADC units as a function of $V_{o p}$. The ordinate axis on the right reports the estimated pixel gain as a function of $V_{o p}$.

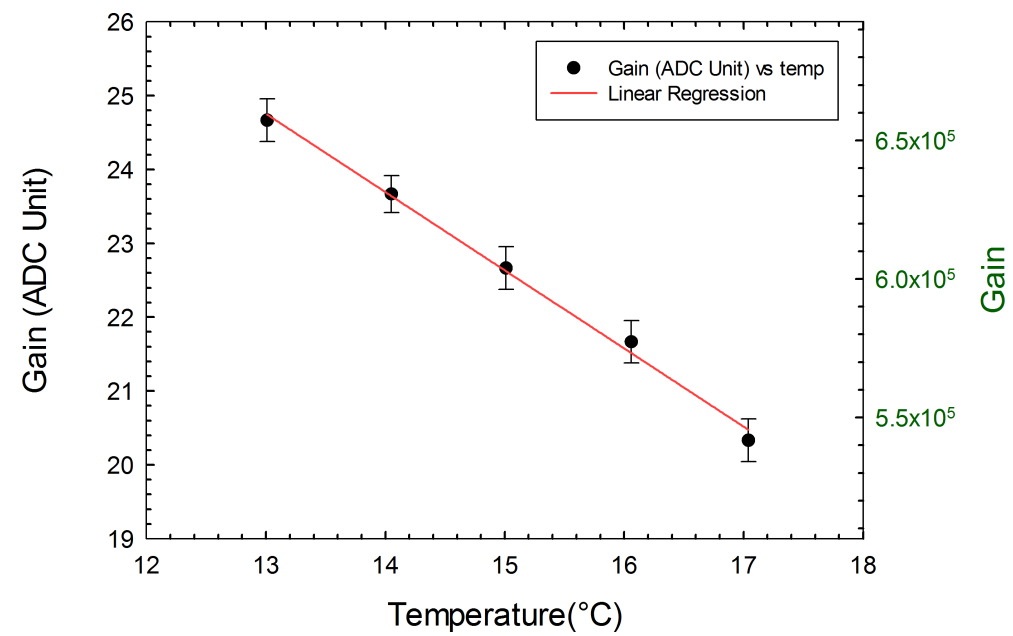

Fig. 10 Determination of the gain variation coefficient in ADC units as a function of temperature. The ordinate axis on the right reports the estimated pixel gain as a function of temperature.

The trigger equalization is determined performing Multiple Fiber Stair MFS measurements after the gain equalization. In this case, the SiPM is illuminated with a pulsed LED that produces a Poissonian distribution with an average number of pe $\sim 4$, and the number of triggers as a function of the discriminator threshold level is collected. The envisaged methodology for trigger equalization is developed in several steps as presented below: 
1. Perform the MFS measurements, setting to zero the 4 bit DAC;

2. Repeat the MFSs, increasing the 4bit DAC code up to 15; Figure 11 shows the trigger rate as a function of the 10bit DAC code for five different 4 bit DAC increments in one of the channel.

3. Differentiate the trigger rate curves to obtain the distribution of the photons emitted by the LED pulses. Figure 12 shows the differential trigger rate obtained from the curves of Figure 11.

4. Compute the average distances in DAC units between adjacent peaks and plot them as a function of the 4 bit DAC code. An increase of the 4 bit DAC corresponds to a shift of the peak

5. Fit the data points with the following equation:

$$
\Delta D A C_{10 b i t s}=m \cdot D A C_{4 b i t s}+q
$$

where $\triangle D A C_{10 b i t s}$ is the average displacement in DAC units between adjacent peaks (see Figure 13). It allows to equalize the signals of all trigger discriminators.

The linear relation between the $10 \mathrm{bit} \mathrm{DAC}$ displacement $\left(\triangle D A C_{10 b i t s}\right)$ and the $4 \mathrm{bit} \mathrm{DAC}$ counts for one of the channels is shown in Figure 12.

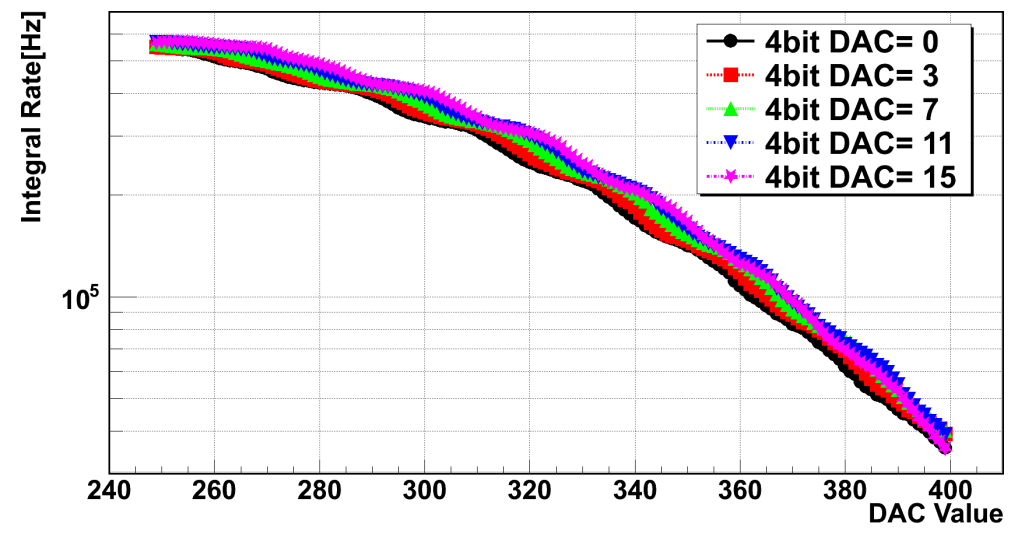

Fig. 11 MFS for one of the channels. Trigger rate as a function of the 10bit DAC code for five increments of the $4 \mathrm{bit} \mathrm{DAC}(0,3,7,11$ and 15$)$. The error of each point is less than 0.1 $\%$, so it is not visible in the figure. 


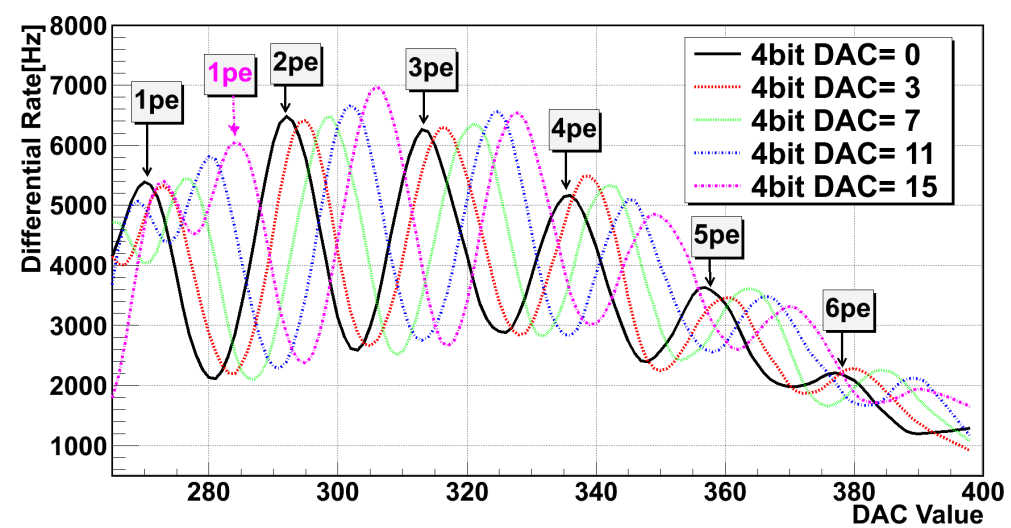

Fig. 12 Differential trigger rate varying the 4 bit DAC in steps of 4 DAC counts. An increase in the $4 \mathrm{bit} \mathrm{DAC}$ code results in a shift of the pe peaks in the abscissa.

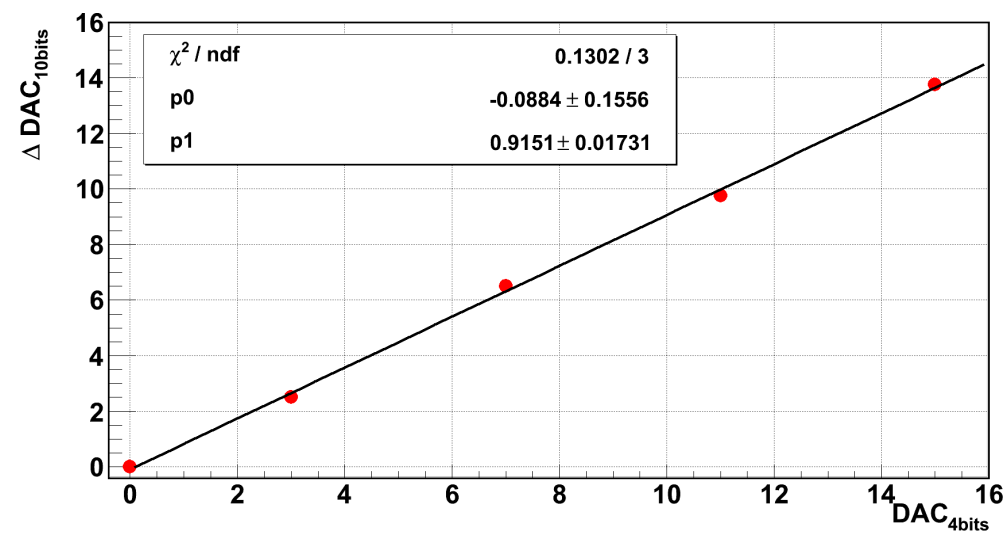

Fig. 13 4bit DAC linearity. The error of each point is $\sim 0.1$ DAC, so it is not visible in the figure. 


\section{Summary and conclusion}

We presented the relative calibration procedures for the ASTRI SST-2M camera designed to keep the gain uniform over the focal plane surface and constant in time. In particular, for each pixel, we determined the reference value of the operative voltage $V_{0}$ with the relative $D A C_{0}$ at temperature of $15^{\circ} \mathrm{C}$ and the reference values of the coefficients $\left(a, b, c_{1}\right.$ and $\left.c_{2}\right)$ used to correct, on field, the variation of the gain due to temperature variations acting on the operative voltage.

The contribution of all error sources, at one $\sigma$ level, to the total gain calibration accuracy, computed for the reference gain $\left(6.0 \times 10^{5}\right)$, are listed in Table 1 . Note that, the errors introduced by the spreads in $\left\langle V_{o p}\right\rangle$ within the camera pixel (see Sect. 5.1) are computed assuming that the spreads at the gain $6.0 \times 10^{5}$ are identical to those measured at $7.5 \times 10^{5}$. This is coherent with our assumption that the value of $c_{1}$ is equal for all pixels (see Sect. 5.3).

Table 1 Contributions to the gain error budget in the calibration procedure. The first column lists the sources of errors whose level is given in the second column; the last column presents the gain error in percentage for the reference gain $6.0 \times 10^{5}$

\begin{tabular}{|l|l|l|}
\hline Error Source & Parameter Error & Percentage \\
\hline SiPM power supply ripple $(\mathrm{mV})$ & \pm 10 & 0.7 \\
\hline 12 bits Pulse Height accuracy $(\mathrm{ADC})$ & \pm 0.1 & 0.5 \\
\hline Temperature sensors $\left({ }^{\circ} \mathrm{C}\right)$ & \pm 0.2 & 0.9 \\
\hline DAC gain adjustment $(8$ bits $)$ & \pm 1 & 1.1 \\
\hline SiPM $<V_{o p}>$ within pixel & $1.1 \times 10^{4}$ & 1.8 \\
\hline$\partial G / \partial V(\mathrm{ADC} / \mathrm{V})$ & \pm 0.78 & 5.3 \\
\hline$\partial G / \partial T\left(\mathrm{ADC} /{ }^{\circ} \mathrm{C}\right)$ & \pm 0.09 & 8.3 \\
\hline
\end{tabular}

We note that our total error budget is $10.1 \%$, compliant with the CTA requirements [18].

In addition, we presented the relative calibration of the trigger necessary to keep uniform the trigger rate on the entire focal plane.

The calibration procedures described in this document are optimized for the current SiPM (Hamamatsu MPPC S11828-3344M), they are still valid for any other SiPM sensors.

\section{Acknowledgements}

Authors thank the anonymous referee for his/her useful comments that really improved the paper. The work presented in this paper was supported in part by the ASTRI, "Flagship Project" financed by the Italian Ministry of Education, University, and Research (MIUR) and led by the Italian National Institute for Astrophysics (INAF). We also acknowledge partial support from MIUR Bando PRIN 2009 and TeChe.it 2014 Special Grants. 


\section{References}

1. T.C. Weekes, et al., "Observation of TeV gamma rays from the Crab nebula using the atmospheric Cerenkov imaging technique", ApJ, 342, p.379-395, (1989).

2. M. Heß, K. Bernlöhr, A. Daum, et al., "The time structure of Cherenkov images generated by TeV $\gamma$-rays and by cosmic rays", Astroparticle Physics 11, 363-377, (1999).

3. Hoffmann W., et al., "The high energy stereoscopic system (HESS) project", Contribution to AIP 515, (1999).

4. Ferenc, D., et al., "The MAGIC gamma-ray observatory", NIM-A, 553, 274-281, (2005).

5. Holder J., et al., "Status of the VERITAS Observatory", Contribution to AIP 1085, (2008).

6. Acharya B. S., et al., "Introducing the CTA concept", Astroparticle Physics 43, 3-18, (2013)

7. N. La Palombara, et al., "The INAF ASTRI Project in the framework of CTA", 14th ICATPP 2013, arXiv:1405.4187 (2014).

8. S. Vercellone et al., "The ASTRI mini-array within the future Cherenkov Telescope Array", Proc. RICAP 2014, EPJ Web of Conferences, EDP Sciences, arXiv: 1508.00799 (2015).

9. The ASTRI Collaboration, Contributions to the 33rd ICRC - arxiv:1307.4639 (2013).

10. G. Pareschi et al., "The dual-mirror Small Size Telescope for the Cherenkov Telescope Array", 33rd ICRC - arxiv:1307.4962 (2013).

11. M. Actis et al., The CTA Consortium, " Design concepts for the Cherenkov Telescope Array CTA: an advanced facility for ground-based high-energy gamma-ray astronomy " Experimental Astronomy, 32, 193-316 (2011), 3.

12. R. Canestrari et al., "The ASTRI SST-2M Prototype: Structure and Mirror", 33rd ICRC - arXiv:1307.4851 (2013).

13. O. Catalano et al., "The camera of the ASTRI SST-2M prototype for the Cherenkov Telescope Array" Proc. SPIE 9147, Ground-based and Airborne Instrumentation for Astronomy V, 91470D (July 8, 2014); doi:10.1117/12.2055099.

14. Hamamatsu, Tech. Rep. KSX-0046 B-2, Hamamatsu Photonics K.K., Solid State Division (2011).

15. Marano, D. et al., "Electro-optical characterization of MPPC detectors for the ASTRI Cherenkov telescope camera", Nuclear Instruments and Methods in Physics Research Section A 768 December (2014), 32.

16. Fleury, J. et al., "Petiroc and Citiroc : Front-end ASICs for SiPM readout and ToF applications", Journal of Instrumentation, Vol. 9, C01049, 2014.

17. Impiombato, D. et al., "Characterization and performance of the ASIC (CITIROC) front-end of the ASTRI camera" Nuclear Instruments and Methods in Physics Research Section A 794 September, (2015), 185.

18. Actis, M., et al., The CTA Consortium . "Design concepts for the Cherenkov Telescope Array CTA: an advanced facility for ground-based high-energy gamma-ray astronomy", Exp. Astron., vol.32, pp.193-316. DOI 10.1007/s10686-011-9247-0 (2011).

19. Buzhan, B. et al., "An advanced study of silicon photomultiplier" ICFA Instrumentation Bulletin 23 (2001) 28-41.

20. Impiombato, D., et al., "Temperature characterization of the CITIROC front-end chip of the ASTRI SST-2M Cherenkov camera", Proc. SPIE 9906, Ground-based and Airborne Telescopes VI, 990645 (July 27, 2016); doi:10.1117/12.2231099 